\title{
Anion exchange method to synthesize layered materials and heterostructures
}

\author{
Rajeev Rai ${ }^{1}$, Ranit Ram $^{2}$ and N Ravishankar ${ }^{3}$ \\ ${ }^{1}$ Indian Institute of Science, Bangalore, Karnataka, India, ${ }^{2}$ Indian Institute of Science, Kharagpur, West Bengal, \\ India, ${ }^{3}$ Indian Institute of Science, United States
}

In recent times, two-dimensional (2D) layered metal dichalcogenides (LMDs) have been extensively studied due to their unique physical and chemical properties and have shown great potential for diverse applications in electronics, ${ }^{1}$ optoelectronic ${ }^{2,3}$ and valleytronic devices, ${ }^{4}$ as well as energy conversion and storage. ${ }^{5}$ Properties of LMDs can be further tailored by stacking or stitching different layers together to form vertical or lateral heterostructure. Exploring effective and controllable approaches to tailor the atomic and subsequently electronic structures of the 2D LMDs via chemical composition is pivotal for facilitating their practical applications and presents a significant challenge in the subject of 2D layered materials.

To date, various approaches such as mechanical exfoliation, liquid exfoliation, chemical vapor deposition (CVD), and wet chemical methods have been explored to synthesize 2D layered materials. However, mechanical exfoliation and CVD methods can produce high-quality mono- or few-layered LMDs but owing to limited scalability, they possess a challenge for practical technological applications. Due to poor-quality control, liquid exfoliation method which can permit a large-scale preparation restricts its useful applications. As a result, it remains an open challenge to grow continuous, high-quality 2D LMDs and their heterostructures in a precisely controllable way. As an alternative method, the solution method has been developed as a low-cost, energy-savvy, bottom-up approach for various colloidal nanocrystals. However, the synthesis of 2D LMDs with respect to size, shape, and composition is still challenging. Post-synthetic methods, one of the wet-chemical methods which has been exploited for synthesizing nanocrystals, can also be employed to synthesize the LMDs and heterostructure based on them. Theoretically, an ion-exchange reaction is strictly controlled by thermodynamic and kinetic parameters. The ion exchange feasibility is governed by the crystal structures of the reactant and product and their relative thermodynamic stabilities.

Herein, using post-synthetic anion- exchange method has been employed to synthesize $\mathrm{SnSe} 2$ by exchanging $\mathrm{S}$ by Se from $\mathrm{SnS2}$, controlled experiments show the process starts to take place from the edges and moves inward. With careful control over the reaction parameter, it is feasible to form lateral heterostructure as well.

Figure 1 shows the microstructure of $\mathrm{SnS} 2$ and anion-exchanged mediated synthesized SnSe2. Figure1(a) shows the low-mag bright-field micrograph of as-synthesized $\mathrm{SnS} 2$ nanosheet. As shown in figure1(b) corresponding diffraction pattern with hexagonal symmetric pattern reveals the single-crystalline nature of the SnS2. Figure 1(d) shows the low-mag bright-field TEM micrograph after the anion exchange, showing the sheet morphology with sharp edges. However, post-exchange the sheets reveal a hole in the center, which may be due to faster diffusion kinetics of $S$ than bigger Se ion. Figure 1(e) shows the corresponding SAED pattern indicating the single-crystalline nature of the sheet after the exchange. Figure 1(f) shows the high-resolution TEM micrograph showing the lattice fringes with the spacing of $3.30 \AA$, which corresponds to (100) plane of SnSe2.

HAADF-STEM images of nanosheets before exchange and after the exchange are shown in figure 2(a and b) revealing the hexagonal arrangement of $\mathrm{S}$ and $\mathrm{Se}$ atoms around $\mathrm{Sn}$ atom. The micrograph has been acquired with $2 \mu$ s per pixel. Mechanistic studies have revealed that the transformation takes place from the edges and moves inward. STEM-EDS map for the intermediate product is shown in figure 2(c) showing the distribution 
of Se, S and Sn. Sn is distributed through out the sheets whereas Se is concentrated on outer side and $\mathrm{S}$ is more on the inner part of the sheets.
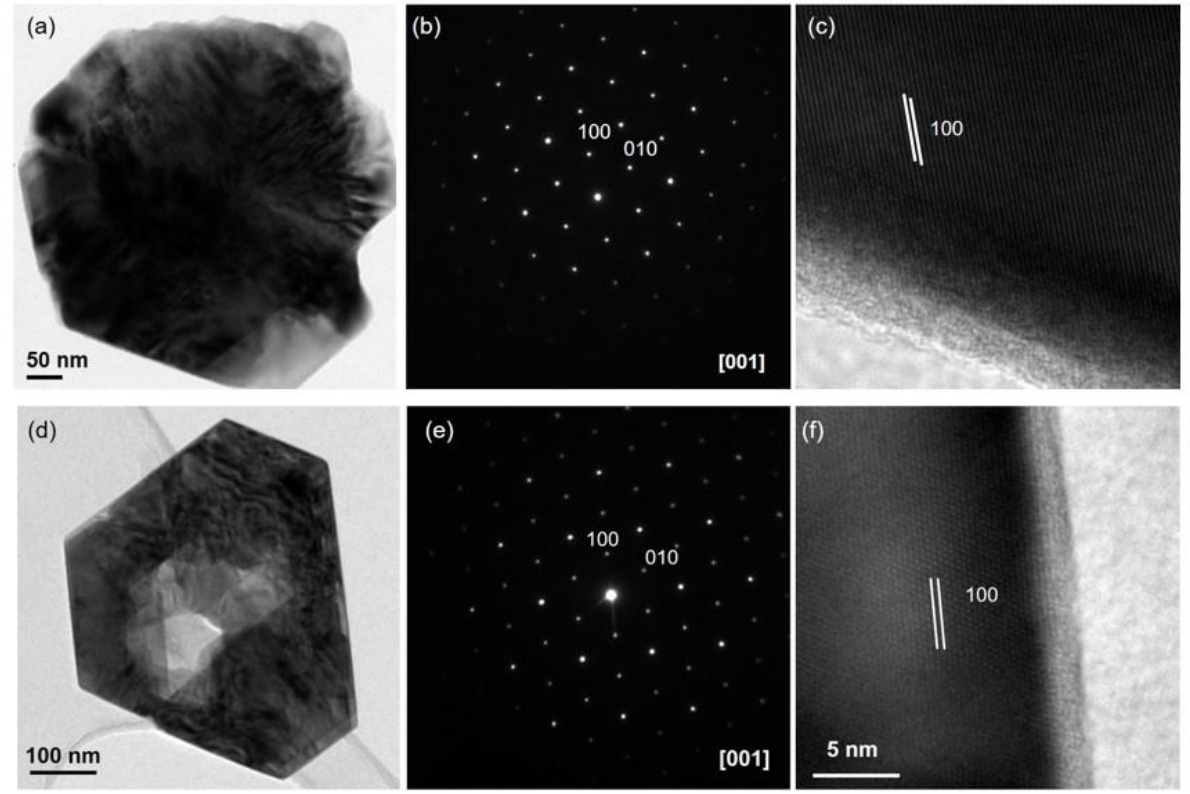

Figure 1. Low-magnification bright-field TEM micrograph, Selected area electron diffraction pattern and High-Resolution TEM micrograph of template SnS2 nanosheets (a, b, and c) and SnSe2 synthesized via the ion-exchange method (d, e, and f) respectively.

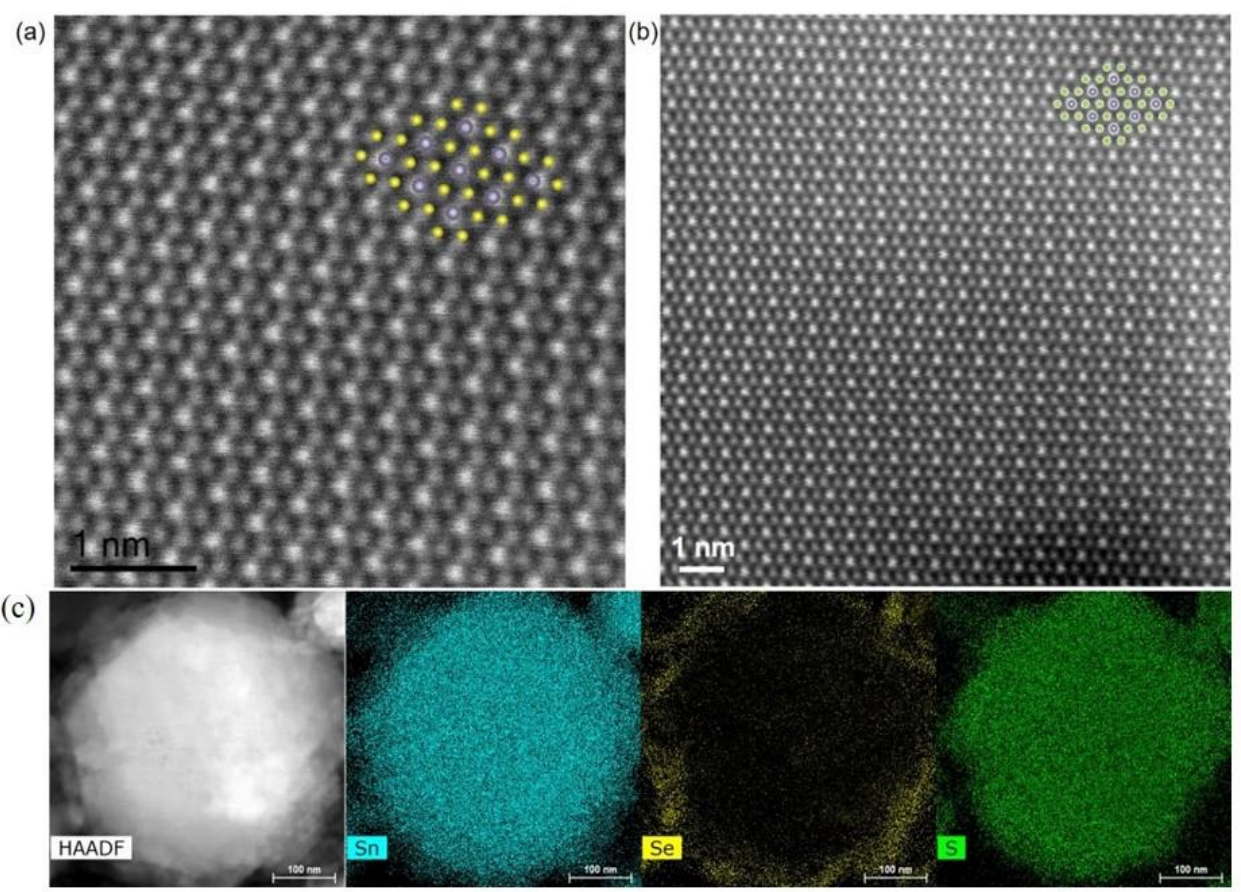


Figure 2. Single frame STEM micrograph acquired at $300 \mathrm{kV}$ showing the atomic arrangement of (a) SnS2; (b) SnSe2 and (c) Low-mag STEM-HAADF micrograph of partial exchanged product and corresponding EDS map showing the elemental distribution of $\mathrm{Sn}, \mathrm{Se}$ and $\mathrm{S}$ respectively.

\section{References}

1. G. Fiori, F. Bonaccorso, G. Iannaccone, T. Palacios, D. Neumaier, A. Seabaugh, S. K. Banerjee and L. Colombo, Nat. Nanotechnol., 2014, 9, $768-779$.

2. Y. Huang, K. Xu, Z. Wang, T. A. Shifa, Q. Wang, F. Wang, C. Jiang and J. He, Nanoscale, 2015, 7, 17375 $-17380$.

3. R. K. Rai, S. Islam, A. Roy, G. Agrawal, A. K. Singh, A. Ghosh, N Ravishankar, Nanoscale, 2019, 11 (3), 870-877.

4. P. Rivera, K. L. Seyler, H. Yu, J. R. Schaibley, J. Yan, D. G. Mandrus, W. Yao, and X. Xu, Science 2016, 351, 688-691.

5. C. Zhang, H. Yin, M. Han, Z. Dai, H. Pang, Y. Zheng, Y.-Q. Lan, J. Bao and J. Zhu, ACS Nano, 2014, 8, 3761-3770. 